\title{
Desarrollo de pensamiento crítico en estudiantes de Fisioterapia, a partir del estudio de las implicaciones sociocientíficas de los xenobióticos
}

\author{
Recibido: 18-11-2010| Aceptado: 30-06-2011
}

Development of critical thinking in students of Physiotherapy, from the social scientific study of the implications of xenobiotics

\section{Nidia Yaneth Torres Merchán*}

\section{Leonardo Fabio Martínez Pérez**}

Resumen: Este artículo presenta los resultados y análisis de una investigación didáctica sobre el desarrollo de algunas habilidades de pensamiento crítico en estudiantes de Fisioterapia que participaron en clases de Bioquímica, centradas en trabajar implicaciones sociocientíficas de los xenobióticos. La metodología de la investigación fue cualitativa estructurada en tres momentos: 1) caracterización de algunas habilidades de pensamiento crítico en los estudiantes 2) estudio de aspectos sociales, ambientales, tecnológicos y bioquímicos involucrados en la cuestión sociocientífica y 3) estructuración con los estudiantes de estudio de casos clínicos. Los datos constituidos en el transcurso de estos momentos favorecieron la enseñanza significativa de conceptos bioquímicos, además de potencializar habilidades de pensamiento crítico.
Palabras clave: Relaciones CTSA, Enseñanza de la Bioquímica, Educación superior, Cuestiones sociocientíficas.

\begin{abstract}
This article presents the results and analysis of educational research on the development of some critical thinking skills in physiotherapy students who participated in Biochemistry classes, focused on social scientific implications of xenobiotics. The research methodology was qualitative structured in three stages: 1) characterization of some critical thinking skills in students 2) study of social, environmental, technological and biochemical aspects involved in social scientific question and 3) structuring of clinical case studies. Data obtained during this time favored the meaningful teaching of biochemical concepts as well as critical thinking skills development.
\end{abstract}

Keywords: CTSA Relations, Biochemistry Teaching, Higher education, social scientific issues.

* Profesora, Universidad Pedagógica y Tecnológica de Tunja, Boyacá - Colombia. Magíster en Docencia de la Química yanethtorres3@hotmail.com

* Profesor Departamento de Química, Universidad Pedagógica Nacional, Bogotá- Colombia. Doctor en Educación para ciencia. Grupo Alternaciencias. lemartinez@pedagogica.edu.co 


\section{Introducción}

Uno de los principales inconvenientes en la enseñanza de las distintas disciplinas es la falta de conexión de los conceptos orientados hacia problemas de la realidad social, por lo que los estudiantes manifiestan aprender distintos tipos de conocimientos sin aplicabilidad alguna a su cotidianidad (Fernández, 2003). Desde esta perspectiva surge la necesidad de promover herramientas de crítica en el aula de clase que tomen algún sentido para los estudiantes. El ejemplo que se pretende ilustrar en este trabajo, como una alternativa pedagógica en esa dirección se refiere a los xenobioticos. En efecto, algunos xenobioticos, en particular, plaguicidas y antibióticos, son usados directa o indirectamente por la sociedad, muchas veces desconociendo sus propiedades bioquímicas y menos reconociendo las implicaciones sociales de su uso, como los efectos sobre los organismos vivos. En ese sentido, problematizar en el aula el uso de estos xenobióticos se establece como una cuestión sociocientifica ${ }^{1}$, que además de llamar la atención del estudiante hacia el aprendizaje de la bioquímica y propiciar

1 El trabajo pedagógico y didáctico basado en cuestiones sociocientíficas abrió un camino concreto para des-marginalizar el enfoque Ciencia, Tecnología, Sociedad y Ambiente (CTSA) del currículo escolar de ciencias (Pedretti, 2003; Martínez, 2010). De esta forma, resulta importante para la formación ciudadana de los estudiantes, comprender la ciencia como una actividad humana que presenta múltiples controversias e incertidumbres en su constitución y, por tanto, requiere de un análisis crítico de sus alcances e impactos en las prácticas de los profesores de ciencias. Así la utilización de cuestiones sociocientíficas, que involucran relaciones CTSA, tiene en cuenta el pensamiento razonado y reflexivo que se centra en decidir qué creer o qué hacer. Éste es un pensamiento de orden superior y como tal, no es automático sino que requiere autodeterminación, reflexión, esfuerzo, autocontrol y metacognición; puesto que en su ejecución se evalúa no sólo el resultado del pensamiento, sino también el proceso mismo del pensamiento. la comprensión de estas temáticas, brinda herramientas críticas frente a los contextos sociales donde se usan.

El profesional de la salud en su desempeño debe identificar y explicar desde su área las alteraciones que se presenten en el organismo, en ese sentido, la enseñanza de la bioquímica a partir de una cuestión sociocientífica, puede aportar tanto elementos básicos para entender el funcionamiento del organismo a partir de las interacciones moleculares como contribuir a la comprensión de implicaciones sociales adyacentes a los casos clínicos estudiados por estos profesionales, dado que este tipo de trabajo pedagógico y didáctico se constituye en una alternativa para la enseñanza de nuevos conocimientos, además de favorecer el desarrollo del pensamiento crítico en los estudiantes.

El trabajo con cuestiones sociocientíficas en la enseñanza de la bioquímica permite que los futuros profesionales se formen como ciudadanos socialmente responsables, mediante la evaluación de las bases que fundamentan su práctica profesional. De acuerdo con lo anterior se tienen en cuenta los planteamientos de Lankshear, Gee y Hull (1996) quienes afirman que el enfoque CTSA, permite desarrollar capacidades científicas que involucran comprensión, curiosidad, creatividad, conciencia crítica del papel de las ciencias en la sociedad, cuidado y disposición responsable. Otros autores tales como Santos y Mortimer (2001) consideran que un sujeto se considera agente responsable cuando acepta un problema social como una preocupación personal y la asume como tal cuando tiene un significado real.

Las cuestiones sociocientíficas en la educación superior permiten procesos de enseñanza y aprendizaje que pueden ser 
Desarrollo de pensamiento crítico en estudiantes de Fisioterapia, a partir del estudio de las implicaciones sociocientíficas de los xenobióticos Nidia Yaneth Torres Merchán | Leonardo Fabio Martínez Pérez

potenciales para que los profesionales en formación, tales como los fisioterapeutas establezcan relaciones entre los aspectos específicos de su profesión con las dimensiones sociales de los mismos, de esta forma nuevas actitudes personales pueden ser promovidas en su campo laboral, por lo que los contenidos deben ser determinados de acuerdo con la práctica profesional. De acuerdo con Zeidler, et al. (2002), las cuestiones sociocientificas, permiten evaluar el desempeño profesional, además de contribuir a la formación de ciudadanos que participen de forma activa y fundamentada en la sociedad. Sadler y Zeidler (2004) afirman que las cuestiones sociocientíficas permiten no sólo asumir papeles de la comunidad científica, sino también asumir roles de la sociedad en general, por lo cual los estudiantes proponen distintas explicaciones a diversos cuestionamientos que les permiten prepararse para enfrentar de manera crítica diversas problemáticas propias del fisioterapeuta. Para Dillon (1994) el abordaje de cualquier disciplina debe considerar elementos controversiales que permitan desarrollar habilidades de argumentación y trabajo cooperativo, además de utilizar una metodología para desarrollar pensamiento crítico e independencia intelectual. Rudduck (1986), afirma que la utilización de cuestiones sociocientíficas hace que los estudiantes enfrenten conflictos, formulen opiniones y tomen decisiones. Stenhouse (1970), manifiesta que el docente debe asumir un papel de dinamizador que formula preguntas, utiliza problemas, mantiene un ritmo interesante en la discusión, promueve la discusión y la autocritica a través de preguntas. Henderson y knutton (1990) confirman que la discusión en grupo, de asuntos controversiales en ciencias permite desenvolver habilidades tales como construir hipótesis, recolectar y analizar datos, fundamentar opiniones, comunicar asertivamente y realizar trabajos cooperativos.
De acuerdo con lo anterior, este trabajo de investigación analizó la solución de problemas y de argumentos, como habilidades de pensamiento crítico en el transcurso de clases de Bioquímica ofrecidas a estudiantes de Fisioterapia, en las cuales se trabajaron las implicaciones sociocientíficas de los xenobióticos. Así, la enseñanza de la bioquímica cobra una importancia en el currículo del fisioterapeuta, en la medida en que este profesional requiere comprender aspectos relacionados con procesos metabólicos y fisiológicos asociados al buen funcionamiento del cuerpo humano. Los xenobióticos pueden ser estudiados como una cuestión sociocientífica y no como un simple tema, ya que de esta forma podemos favorecer la estructuración de un ambiente pedagógico-didáctico que contribuya con la formación de pensadores críticos, que en términos de Facione (1998) implicaría el desarrollo de personas habitualmente indagadoras, diligentes en la búsqueda de información relevante, razonables en la selección de criterios y persistentes en la búsqueda de resultados pertinentes como las circunstancias o el problema lo permitan. Todo ésto implica cuestionar la trasmisión de contenidos que no considera sus implicaciones sociales, lo que es relevante para la formación profesional del fisioterapeuta, ya que él trabaja en el área de la salud enfrentando problemas de orden social relacionados con la calidad de vida de los pacientes.

\section{Metodología}

La investigación adoptó una metodología cualitativa en la medida en que se concibe la constitución de datos como un proceso social y subjetivo, en el cual las acciones de los investigadores involucran intenciones y valores que influyen tanto en el trabajo de campo desarrollado como en los correspondientes análisis. 
La investigación cualitativa implica un acercamiento cuidadoso del investigador al contexto educativo en el que desarrollará su estudio, de tal manera que se construyen un conjunto de prácticas materiales interpretativas, a través de las cuales se busca la comprensión de los problemas estudiados. De acuerdo con Denzin y Lincoln (2006) esas prácticas se materializan y representan la situación estudiada por medio de diversos registros cualitativos. Aunque la investigación fue cualitativa se articularon elementos cuantitativos para caracterizar algunas habilidades de pensamiento crítico de los estudiantes, el resto de los análisis fueron cualitativos en términos de discutir las implicaciones sociocientíficas de los xenobióticos y el desarrollo de habilidades de pensamiento crítico en los estudiantes participantes de la investigación.

En el estudio participaron 53 estudiantes de Fisioterapia de una universidad particular de Colombia. Las edades de los estudiantes oscilaban entre los 17 a 22 años y todos participaban de la clase de Bioquímica.

Con el objetivo de analizar la solución de problemas y el análisis de argumento, como habilidades de pensamiento crítico en el transcurso de clases de Bioquímica ofrecidas a estudiantes participantes de la investigación se estructuraron los siguientes momentos metodológicos:

- Caracterización de la habilidad de los estudiantes para solucionar problemas y analizar argumentos, utilizando el test de Halpern para la evaluación del pensamiento crítico mediante situaciones cotidianas (HCTAES) (Halpern, 1998).

- Estudio de aspectos sociales, ambientales, tecnológicos y bioquímicos involucrados en la cuestión de los xenobióticos, a partir de artículos presentados en medios de comunicación.
- Estructuración con los estudiantes de estudio de casos clínicos que aborden la cuestión de los xenobióticos, en la perspectiva de desarrollar habilidades de pensamiento crítico.

Estos momentos fueron desarrollados durante 5 sesiones de clase, cada una con una duración de 2 horas y 60 minutos, las cuales fueron grabadas en audio para la constitución de datos.

Se realizó un análisis verbal de los datos, que permitiera hacer una reelaboración de los conceptos de enseñanza y de aprendizaje en los estudiantes y la docente. Las grabaciones se efectuaron por grupos de trabajo en cada una de las sesiones planeadas. Los resultados y análisis de cada uno de los momentos expuestos anteriormente son descritos a continuación.

\section{Caracterización inicial de habilidades de pensamiento crítico}

Para realizar una caracterización inicial de habilidades de pensamiento crítico en los estudiantes de Fisioterapia se aplicó el test de Halpern (1998), que permitió la evaluación del pensamiento crítico mediante situaciones cotidianas. Delimitamos el estudio al análisis de las habilidades de argumentación y solución de problemas, considerando que estas dos habilidades son importantes para que los estudiantes manifiesten sus opiniones, explicaciones y solucionen problemas relacionados con la Bioquímica. El desarrollo de habilidades de argumentación y solución de problemas permite que el proceso de enseñanza aprendizaje se convierta en un proceso cultural y comunicativo que implica la negociación de significados, ya que el 
Desarrollo de pensamiento crítico en estudiantes de Fisioterapia, a partir del estudio de las implicaciones sociocientíficas de los xenobióticos Nidia Yaneth Torres Merchán | Leonardo Fabio Martínez Pérez

significado no es preexistente sino que se construye durante la comunicación y surge de la interacción (Gómez, 2000).

Para el análisis de las habilidades de pensamiento crítico se utilizaron 5 preguntas (Anexo 1) ${ }^{2}$ con formato doble, lo que quiere decir que primero se presenta una pregunta abierta en la que el sujeto debe ofrecer un argumento, o una explicación, o generar las soluciones a un problema, u opinar sobre algo y tras ello se muestra una pregunta cerrada en la que el sujeto debe elegir entre una serie de alternativas aquella que mejor responda o resuelva el problema. Según Halpern (1998) este doble formato de preguntas permite conocer por un lado, si el que responde al test manifiesta un uso espontáneo de la habilidad y por otro, si es capaz de usarla cuando se le señala que es necesaria para esa situación, aún cuando espontáneamente no haya reconocido que fuera necesaria.

En la tabla 1, describimos las características de las habilidades de pensamiento crítico valoradas en este estudio.

\begin{tabular}{|c|l|}
\hline Habilidades & \multicolumn{1}{c|}{ Características } \\
\hline $\begin{array}{c}\text { Análisis de } \\
\text { argumento }\end{array}$ & $\begin{array}{l}\text { Las habilidades de razonamiento verbal y análisis de argumento } \\
\text { permiten identificar y valorar la calidad de las ideas, razones de } \\
\text { un argumento y la conclusión del mismo. Además, permiten } \\
\text { reconocer analogías dentro del lenguaje cotidiano. }\end{array}$ \\
\hline $\begin{array}{l}\text { Solución de } \\
\text { problemas }\end{array}$ & $\begin{array}{l}\text { Esta habilidad permite ejercitar las habilidades de razonamiento } \\
\text { en el reconocimiento y definición de un problema a partir de } \\
\text { ciertos datos, en la selección de la información relevante y la } \\
\text { contrastación de las diferentes alternativas de solución y de sus } \\
\text { resultados. Expresar un problema en formas distintas y generar } \\
\text { soluciones. }\end{array}$ \\
\hline
\end{tabular}

Tabla 1. Características de habilidades de pensamiento crítico analizadas.

Los datos señalados en la figura 1, corresponden a la tabulación de los resultados del test HCTAES obtenidos en la prueba diagnóstica que permitieron identificar las habilidades de pensamiento crítico (análisis de argumento y solución de problemas) en los estudiantes participantes del estudio. Esta figura señala que la mayoría de los estudiantes obtienen un valor entre el 40 y $60 \%$ en la solución de problemas, 20 estudiantes alcanzan un promedio entre el 60 y $80 \%$ en las dos habilidades, menos de 5 estudiantes obtienen valores entre el 80 y $100 \%$ ninguno de los estudiantes obtienen valores menores al $10 \%$.

2 En el anexo presentamos cuatro partes del instrumento aplicado, fueron excluidas 6 partes del anexo por cuestiones de espacio, no obstante las otras partes del instrumento tienen la misma estructura que las partes presentadas. 


\section{Comparación de habilidades evaluadas con el test HCTAES}

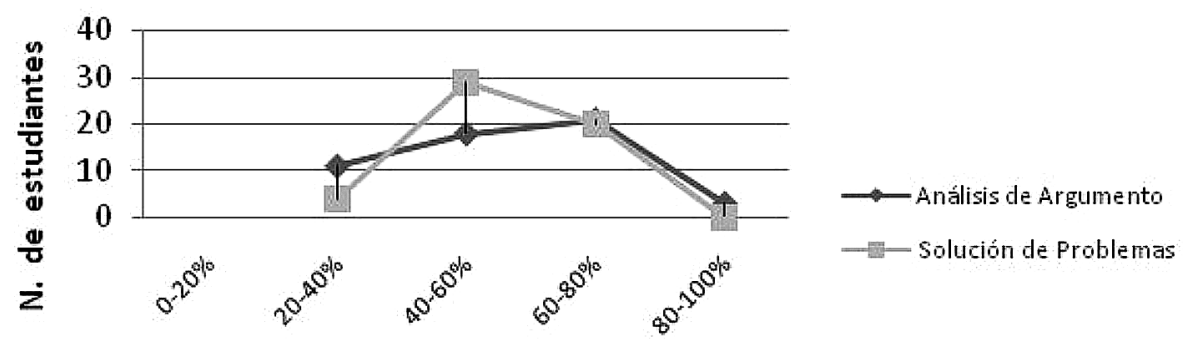

Porcentaje en cada Habilidad

Figura 1. Porcentaje de análisis de argumento vs. solución de problemas

\section{Niveles de Pensamiento Crítico}

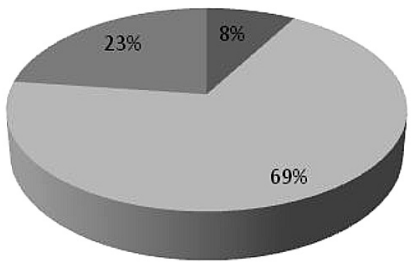

\footnotetext{
- Nivel B Pensamiento crítico entre el $21-40 \%$

- Nivel C Pensamiento crítico entre el $41-60 \%$

- Nivel D Pensamiento crítico entre el $61-80 \%$
}

Figura 2. Niveles de pensamiento crítico en relación con las habilidades evaluadas.

La figura 2, permite observar cómo se encuentran los estudiantes caracterizados de acuerdo con las habilidades de interés. El $69 \%$ de la población estudiada posee un pensamiento crítico en el nivel $C$, es decir menor al $60 \%$, lo que permite inferir el desarrollo de estrategias metodológicas para potencializar estas habilidades.

De acuerdo con los resultados obtenidos en la caracterización de habilidades podemos afirmar que los estudiantes de Fisioterapia poseen habilidades de pensamiento crítico (análisis de argumento y solución de problemas), pero no están potencializadas, lo que requiere de estrategias que permita potencializar pedagógicamente en mayor rango habilidades como: análisis de argumento y solución de problemas. Esta caracterización previa sugiere que los docentes conviertan las aulas educativas en escenarios de aprendizaje colectivo favoreciendo mejores 
Desarrollo de pensamiento crítico en estudiantes de Fisioterapia, a partir del estudio de las implicaciones sociocientíficas de los xenobióticos Nidia Yaneth Torres Merchán | Leonardo Fabio Martínez Pérez

condiciones y oportunidades para desarrollar habilidades de pensamiento crítico que permitan ejercitar el análisis de argumento y la solución de problemas.

Considerando los resultados arrojados por el test HCTAES se suministraron lecturas a los estudiantes para conceptualizar algunos aspectos de los xenobióticos, al igual que para evidenciar algunas de sus implicaciones positivas y negativas en la salud. A partir de estas lecturas se buscó articular conceptos bioquímicos y aspectos de la salud, favoreciendo un ambiente educativo que posibilitara en los estudiantes su participación, así como la estructuración de puntos de vista propios y diálogo con otros puntos de vista, evitando de esta manera una actuación pasiva que reduce los estudiantes a receptores de respuestas correctas del docente.

Como trabajo complementario se programaron sesiones de asesoría en pequeños grupos respecto a las actividades suministradas, estas asesorías permitieron que los estudiantes buscaran la orientación y aclaración de ciertos términos y comprendieran la acción de los xenobióticos en el organismo estableciendo diversas relaciones entre los conceptos disciplinares.

En las actividades descritas se dio gran importancia al diálogo, permitiendo que los estudiantes interpretaran textos, analizaran dificultades, relacionaran la información suministrada y se elaborara un trabajo colectivo a partir de cuestiones relacionadas con su profesión. Todas estas actividades posibilitaron que los estudiantes actuaran como pensadores activos que participan, defienden opiniones, escuchan y argumentan.

También se realizó una síntesis de las consideraciones planteadas por los estudiantes acerca de la cuestión sociocientífica estudiada. En relación con lo anterior el 15 $\%$ de los estudiantes consideró que la síntesis de productos en los laboratorios solamente se da por intereses económicos que permiten aumentar los ingresos de quienes los producen, sin pensar en las consecuencias que éstos puedan traer al ambiente. Un $30 \%$ defiende la síntesis de sustancias químicas para la erradicación de enfermedades y el $55 \%$ de los estudiantes está de acuerdo en que es importante la síntesis de sustancias químicas pero realizando estudios previos para determinar sus posibles efectos en la sociedad. Esta primera parte permitió hacer también un reconocimiento de la ciencia como una ciencia que posee limitaciones, responsabilidades, así como involucra aspectos, políticos, económicos, culturales y ambientales.

En relación con los aspectos positivos y negativos de los xenobióticos en la salud, el $15 \%$ de los estudiantes consideró que la síntesis de productos en los laboratorios solamente se da por intereses económicos que permiten aumentar los ingresos de quienes los producen, sin pensar en las consecuencias que éstos puedan traer al ambiente. Un 30 \% defendió, la síntesis de sustancias químicas para la erradicación de enfermedades y un $55 \%$ de los estudiantes estuvo de acuerdo en que es importante la síntesis de sustancias químicas, pero realizando estudios previos para determinar sus posibles efectos en la sociedad.

\section{Estudio de casos clínicos a partir del abordaje de cuestiones sociocientíficas en la enseñanza de la bioquímica}

Para potencializar la habilidad de solución de problemas se trabajó con los estudiantes el caso clínico del envenenamiento causado por el cianuro. Este caso permitía evaluar 
la habilidad de solución de problemas y el análisis de argumentos para dar paso a actividades centradas en aspectos bioquímicos, sociales y ambientales de los xenobióticos.

En la tabla 2, se presentan los tópicos abordados en el caso clínico utilizado. Con respecto a las Benzodiacetinas se com- prendió el efecto de estos xenobióticos como medicamentos psicotrópicos que actúan sobre el sistema nervioso central, con efectos sedantes y relajantes, utilizados para tratar los estados de pánico causadas por las intoxicaciones por alucinógenos que circulan en la sangre unida a proteínas plasmáticas.

\begin{tabular}{|l|l|}
\hline \multirow{4}{*}{$\begin{array}{c}\text { Sistemas } \\
\text { energeticos }\end{array}$} & La acidosis y alcalosis metabólica y respiratoria \\
\cline { 2 - 2 } & Sistemas anaerobio láctico, anaeróbico aláctico, sistema aerobio \\
\cline { 2 - 2 } & Importancia del ATP \\
\cline { 2 - 2 } & Efecto del nitrito de amilo \\
\cline { 2 - 2 } & Efecto de la benzodiacetinas \\
\cline { 2 - 2 } & Efecto del midazolan \\
\hline
\end{tabular}

Tabla 2. Tópicos abordados desde el estudio del caso clínico: "Envenenamiento por Cianuro".

Con la discusión del caso clínico los estudiantes comprendieron los procesos a través de los cuales el cianuro causa daño cerebro vascular, explicando sus efectos de la siguiente manera:

\section{Estudiante (E)}

$E_{1}$. El daño cerebro - vascular causado por cianuro, resulta de la combinación del ácido cianhídrico con otros compuestos, este compuesto se une a la citocromo-oxidasa e inhibe la fosforilación del ATP, como en la cadena respiratoria hay hierro, que actúa como facilitador, se une al hierro bloqueando la cadena respiratoria ocasionado: acidosis metabólica.

$E_{2}$. El cianuro inhibe la cadena respiratoria, ocasionando que la paciente tenga inconvenientes en los sistemas energéticos porque sin cadena respiratoria no se puede producir ATP.

$E_{3}$. Como parte del tratamiento (frente a la intoxicación con cianuro) se administra nitritos que convierten la hemoglobina en metahemoglobina que tiene mayor afinidad por el cianuro que la citocromo oxidasa, permitiendo que este se pueda eliminar en forma de tiocianato por la orina. 
Desarrollo de pensamiento crítico en estudiantes de Fisioterapia, a partir del estudio de las implicaciones sociocientíficas de los xenobióticos Nidia Yaneth Torres Merchán | Leonardo Fabio Martínez Pérez

El abordaje de las características bioquímicas de los xenobióticos entre los estudiantes y la docente, permitió comprender como estos compuestos fueron utilizados para determinar la secuencia de transportadores de electrones en la cadena de transferencia de electrones en la mitocondria.

Frente al primer xenobiótico (dinitrofenol) estudiado en el caso clínico, se comprendió que actúa como acoplante químico que no afecta la estructura mitocondrial, constituye un acido débil con propiedades hidrófobas, lo que le permite difundirse a través de la membrana de la mitocondria.

Frente al segundo xenobiótico estudiado (oligomicina) se comprendió que actúa como un inhibidor del ATP sintetasa en el complejo V. Este xenobiótico es usado con drogas y sustancias químicas administradas por vía exógena para sus efectos en tejidos vivos y organismos. Incluye aceleración e inhibición de los procesos fisiológicos y bioquímicos y otros mecanismos de acción farmacológica.

El trabajo desarrollado sobre los xenobióticos en el caso clínico facilitó un trabajo investigativo de todos los estudiantes para explicar el efecto de estos xenobióticos dentro de la célula, esto constituye una herramienta importante para considerar al estudiantado como agentes capaces de aprender y mejorar, proponiéndose nuevos límites. Además, permite reestructurar la práctica docente de tal forma que privilegie la participación de los estudiantes; así, es posible que el lenguaje de las ciencias se aprenda a medida que se van entendiendo las formas científicas de ver los procesos y de pensar sobre ellos. Por eso una manera de aprender el lenguaje científico es pensar, hablar, leer y escribir ciencias (Izquierdo y Sanmartí, 2000).

\section{Impacto de los xenobioticos a nivel socioambiental}

En cuanto a los aspectos ambientales, sociales y tecnológicos trabajados en el caso clínico descrito anteriormente, los estudiantes identificaron los aspectos expuestos en la tabla 3 (Ver tabla 3).

Los aspectos identificados por los estudiantes e ilustrados en la tabla 3 facilitaron la comprensión de los temas trabajados en el caso clínico y posibilitaron el abordaje de algunas de sus implicaciones sociales y ambientales.

\section{Estructuración con los estudiantes de estudio de casos clínicos que abordan la cuestión de los xenobióticos, en la perspectiva de desarrollar habilidades de pensamiento crítico}

En este punto los estudiantes trabajaron diversos casos clínicos que involucraron las fumigaciones con el glifosato, y que articulaban aspectos socioambientales de la cuestión sociocientífica con conceptos de Bioquímica. Los casos clínicos utilizados permitieron potencializar la habilidad para solucionar problemas y el análisis de argumento, contribuyendo a la preparación del futuro fisioterapeuta.

La primera habilidad se evaluó de acuerdo con las respuestas que los estudiantes daban a los diferentes cuestionamientos hechos en cada uno de los casos, la segunda permitió vincular la realidad con los conceptos bioquímicos trabajados, lo que contribuyó a la formación de actitudes favorables al campo profesional de los estudiantes. 


\begin{tabular}{|c|c|c|c|}
\hline & $\begin{array}{c}\text { Aspectos } \\
\text { ambientales }\end{array}$ & $\begin{array}{l}\text { Aspectos } \\
\text { sociales }\end{array}$ & $\begin{array}{c}\text { Aspectos } \\
\text { tecnológicos }\end{array}$ \\
\hline Positivos & $\begin{array}{l}\text { “El empleo de } \\
\text { fármacos y otros } \\
\text { productos químicos } \\
\text { (xenobióticos) que } \\
\text { se incorporan a las } \\
\text { granjas con fines } \\
\text { sanitarios para } \\
\text { mejorar el ren- } \\
\text { dimiento animal } \\
\text { vegetal". }\end{array}$ & $\begin{array}{l}\text { "Permiten el trata- } \\
\text { miento de enferme- } \\
\text { dades que afectan a } \\
\text { la población". }\end{array}$ & $\begin{array}{l}\text { "Permiten el desarro- } \\
\text { Ilo de nuevos mate- } \\
\text { riales como plásticos, } \\
\text { medicamentos y } \\
\text { plaguicidas". }\end{array}$ \\
\hline Negativos & $\begin{array}{l}\text { "Los xenobióticos } \\
\text { generan residuos } \\
\text { tóxicos que producen } \\
\text { contaminación al } \\
\text { ambiente, generán- } \\
\text { dose alteraciones en } \\
\text { el equilibrio ecoló- } \\
\text { gico". }\end{array}$ & $\begin{array}{l}\text { "Los xenobióticos } \\
\text { legalmente } \\
\text { permitidos pueden } \\
\text { ser } \\
\text { nocivos si se emplean } \\
\text { a dosis superiores } \\
\text { a las autorizadas o } \\
\text { si no se respetan } \\
\text { sus periodos de } \\
\text { suspensión" }\end{array}$ & $\begin{array}{l}\text { "La acumulación } \\
\text { de muchos de los } \\
\text { productos fabrica- } \\
\text { dos generan efectos } \\
\text { negativos en la salud". }\end{array}$ \\
\hline
\end{tabular}

Tabla 3. Aspectos ambientales, sociales, tecnológicos abordados por los estudiantes.

La habilidad para solucionar problemas según Saiz y Nieto (2002) es de alto nivel en el desarrollo del pensamiento crítico porque los estudiantes deben debatir, argumentar, evaluar, juzgar y criticar, utilizando otras habilidades como: razonamiento verbal, análisis de argumento, incertidumbre y formulación de hipótesis.

El esquema trabajado con los estudiantes para abordar los casos clínicos y solucionar sus correspondientes problemas fue el sugerido por Kortland (1996), partiendo de la identificación de problemas prosiguiendo con el establecimiento de criterios para la evaluación de alternativas, establecimiento de soluciones y concluyendo con el monitoreo de sus implicaciones. Utilizando estos puntos para el estudio de los problemas abarcados por los casos clínicos los estudiantes fueron constituyendo las siguientes alternativas de acuerdo con los síntomas, los aspectos toxicológicos y la historia clínica del caso considerado.

Un 55\% de los estudiantes tuvo en cuenta aspectos generales sobre los xenobióticos resaltando sus características, efectos, mecanismo de acción y resultados de exámenes de sangre y orina. El siguiente fragmento discursivo de un estudiante ilustra la forma como se avanzó en los aspectos descritos anteriormente: 
Desarrollo de pensamiento crítico en estudiantes de Fisioterapia, a partir del estudio de las implicaciones sociocientíficas de los xenobióticos Nidia Yaneth Torres Merchán | Leonardo Fabio Martínez Pérez

\begin{abstract}
$E_{1}:$ Pienso que es importante mirar si hay intoxicación por medicamentos, metales, disolventes, gases, plaguicidas, productos fitosanitarios, fertilizantes, reactivos químicos, los cuales pueden ocasionar en el paciente cuadros clínicos graves a nivel respiratorio y muchas afecciones que pueden provocar los xenobióticos en un organismo celular y provocar la muerte del paciente.
\end{abstract}

Un 25\% de los estudiantes tuvo en cuenta síntomas, vías de administración, clase y peligrosidad del tóxico, tiempo transcurrido desde su uso y evolución del paciente:

$E_{4}:$ Tendría en cuenta antecedentes del paciente, vías de administración, clase y peligrosidad del tóxico, tiempo transcurrido desde su uso, indagaría sobre el tratamiento a seguir de acuerdo con la toxicidad y verificaría si corresponden a los síntomas que presenta el paciente.

El 20\% restante de los estudiantes averiguó sobre la actividad laboral del paciente y antecedentes de intoxicación por determinados fungicidas.

$E_{5}$ : Si es el caso que el paciente ingresa por intoxicación con productos químicos como resultado de su actividad laboral, remitiría a la trabajadora social para tener claridad sobre las exposiciones con las que trabaja el paciente, y en dado caso indagaría sobre jornadas de capacitación frente al manejo de estos productos, es necesario tener en cuenta el plan de salud ocupacional que se lleva a cabo en esta empresa.

A partir de las expresiones de los estudiantes y las experiencias desarrolladas con ellos durante las clases, percibimos que cuando se asume un problema real en la enseñanza de la bioquímica, en este caso el envenenamiento por cianuro, se favorece un diálogo entre docente y estudiantes que facilita la compren- sión de los temas, se promueve un esfuerzo intelectual por parte de los estudiantes para consultar previamente algunos conceptos y articular diversos términos.

También, se observa el desarrollado de valoraciones de los estudiantes que muestran indicios de una comprensión diferente de la bioquímica vinculada al análisis de ventajas y desventajas de la producción de xenobióticos que involucra aspectos socioambientales. Así mismo, en el trabajo desarrollado, los estudiantes se motivaron sobre el estudio de la materia por tener la oportunidad de manifestar sus puntos de vista una cuestión sociocientífica que promueve la construcción de significados compartidos. También se avanzó en superar la idea de la clase de bioquímica como una asignatura de repetición memorística de contenidos específicos.

Evidenciaremos estos avances de mejor forma utilizando los datos constituidos a partir de una entrevista realizada a los estudiantes al final del trabajo desarrollado (Anexo 2), estos datos serán en algunas ocasiones comparados con expresiones que ellos planteaban al iniciar la asignatura de bioquímica.

De acuerdo con las expresiones constituidas en la tabla 4, podemos decir que los estudiantes manifestaron una mejor disposición frente al aprendizaje de conceptos bioquímicos, asumieron la asignatura como importante en su desempeño profesional. También afirmaron que el trabajo realizado a partir de la cuestión sociocientífica se convierte en una oportunidad para comprender la bioquímica como una ciencia relacionada con aspectos de la vida, reconociendo la necesidad de construir un ambiente favorable para el aprendizaje colectivo, en el cual se motiva a investigar sobre temas relacionados con su profesión. A su vez, los estudiantes reconocen la importancia del trabajo colectivo en la comprensión de 
diversos conceptos, además de generar un ambiente participativo para la mayor parte de miembros del grupo.

También preguntamos a los estudiantes si evidenciaban la vinculación de los xenobióticos en la orientación de la clase de bioquímica. Un 57\% de los estudiantes contestaron que esta vinculación se evidenciaba en discusiones sobre la síntesis de productos en un laboratorio, mecanismos de acción de los medicamentos en el organismo y efectos de los xenobióticos en los ecosistemas.

Otros estudiantes afirmaron que se articularon los tópicos por medio de casos clínicos, se realizaron revisiones bibliográficas con orientaciones de la docente, se discutieron ventajas y desventajas de los xenobióticos $y$ efectos que pueden traer al ambiente.

La mayoría de los estudiantes también coincidió en señalar que la clase de bioquímica mejoró al involucrar problemas reales en los casos clínicos estudiados, esto permitió reconocer que en la asignatura se seguía una orientación disciplinar sin tener cierta articulación con problemas que sin duda alguna facilitaban la comprensión de la disciplina. Este trabajo permitió pasar de un modelo tradicional que se venía dando a un modelo alternativo, donde docentes y estudiantes son sujetos activos en el proceso de aprendizaje. (Ver tabla 4)

Con respecto a las opiniones que los estudiantes manifestaron de la bioquímica en su preparación como futuros fisioterapeutas, la mayoría de estudiantes reconoció la importancia de ésta en el desarrollo profesional y estuvo de acuerdo con las siguientes respuestas:

"Es una asignatura interesante ya que proporciona bases para entender diversas patologías, permite comprender diversos procesos bioquímicos en nuestro organismo. "Proporciona nuevos conocimientos, para el desempeño profesional". "Es una clase interesante ya que los conceptos químicos se hacen aplicables a lo biológico". "Es una clase donde hay interacción docente estudiantes, que facilita un mejor entendimiento de los conceptos explicados". Me parece interesante ya que es una materia que reúne conocimientos de otras ciencias y permite estudiar los componentes de los seres vivos como lípidos, proteínas etc.". "Estas clases son importantes pues nos brindan el conocimiento necesario acerca de las reacciones de nuestro cuerpo y nos ayuda a comprender fenómenos, relacionados con nuestra carrera".

Por lo anterior, la utilización de cuestiones sociocientíficas fue una estrategia de razonamiento que permitió a los estudiantes utilizar y potencializar habilidades de pensamiento crítico (análisis de argumento y solución de problemas). También se evidenció la utilización de estrategias significativas y diferentes a las basadas en métodos trasmisionistas de contenidos. Las nuevas estrategias preparan a los estudiantes en la manera de enfrentar situaciones problemas propias de su profesión, se hace conveniente partir de las opiniones que los alumnos tienen acerca de temáticas en una disciplina y la manera como actúan frente a la solución de problemáticas controversiales.

Los estudiantes aumentaron su interés por el estudio de esta disciplina dado que analizan la importancia de la bioquímica como una herramienta importante para entender procesos de la vida que permite explicar el efecto de sustancias en la sociedad y el ambiente, es así como las cuestiones sociocientíficas contribuyen con el desarrollo de una racionalidad autónoma y critica en los estudiantes sobre las comprensiones que poseen frente al mundo 
Desarrollo de pensamiento crítico en estudiantes de Fisioterapia, a partir del estudio de las implicaciones sociocientíficas de los xenobióticos Nidia Yaneth Torres Merchán | Leonardo Fabio Martínez Pérez

\section{Momentos en que se registró la expresión de los estudiantes}

\section{Comienzo del trabajo con la cuestión sociocientífica de los xenobióticos}

Finalización del trabajo desarrollado con la cuestión sociocientífica de los xenobióticos.

- "Se hace explicación magistral seguida de evaluaciones, durante cada sesión se hacían preguntas". "La metodología era llegar a clase, tener atención, aclarar dudas, hacer ejercicios, consultar." "Hay explicación de temas bioquímicos donde se sigue el plan de estudios"

- "La clase involucraba solo aspectos de bioquímica, no había un dialogo más directo con la docente ni los compañeros"." "Es una clase donde casi no hay interacción docente estudiantes que facilitara un mejor entendimiento de los conceptos explicados".

- "Casi no participábamos en clase".

- "El trabajo en clase siempre es igual".
- "Se construye el conocimiento entre todos porque se conocen las opiniones de todos". "Fue un trabajo excelente, ya que tienen mucha relación con los temas de clase". "Este tema contribuyó a mi formación profesional, ya que son conceptos nuevos que se requieren saber en el campo de la fisioterapia"." Fue un tema muy interesante permitió analizar los efectos positivos y negativos de los xenobióticos". "Este tipo de trabajo nos permitió adquirir nuevos conocimientos, puesto que estamos expuestos a dar criterios propios sobre cualquier tema".

- "Pienso que el trabajo fue bueno y didáctico me motivo a interesarme más por la asignatura, dado que cambió porque la profesora permitió mayor participación de nosotros". “Es una metodología extraña porque todos podíamos expresar lo que considerábamos, y esto permitió defender nuestros criterios que eran respetados". "Permitió investigar, leer para empaparnos bien del tema y dar opiniones valederas".

- "Fue un trabajo que permitió que el grupo se uniera más y se conociera y permitió la participación de los compañeros que no participaban".

- "El trabajo que se realizó fue creativo e interesante ya que al discutir entre nosotros mismos una problemática dio puntos de vistas diferentes que nos hicieron proponer y discutir aspectos que nos llevan a una conclusión final así como también a proponer soluciones".

Tabla 4. Expresiones de los estudiantes en diferentes momentos del trabajo desarrollado con cuestiones sociocientíficas.

que los rodea. De esta forma, el desarrollo de pensamiento crítico es un proceso que permite que los estudiantes construyan una visión más amplia del mundo apoyados en espacios de discusión que permiten la construcción de conocimientos a partir de discusiones colectivas.

En este sentido, el análisis de argumentos a través de cuestiones controvertidas como los xenobióticos resulta un aspecto fundamental para la justificación de puntos de vista propios de los estudiantes, de manera que tienen mayores posibilidades de participar crítica y responsablemente en la solución de problemas.

Se requiere proporcionar a los estudiantes situaciones problema poco delimitadas y transversales que permitan realizar una reflexión profunda en la formación de valores, así como de acciones acordes con 
las necesidades del momento actual. Así, el pensador crítico no sólo adquiere los contenidos mismos de la disciplina, sino que también aprende a solucionar problemas y tomar decisiones.

Considerando la cantidad de información que se puede abordar en disciplinas como la bioquímica se hace necesario disponer de habilidades de pensamiento crítico que le permitan valorar y elaborar argumentos, percibir y reconocer variaciones, analizar relaciones causales, disponer de buenas estrategias de toma de decisiones, poseer recursos para resolver ciertos problemas, etc. Se debe crear, además un ambiente de motivación que propicie el desarrollo de pensamiento crítico, dado que este tipo de pensamiento depende en gran medida de las disposiciones por lo que se hace necesario incorporar en el proceso de enseñanza situaciones llamativas que tengan sentido para los estudiantes.

Es importante formar futuros profesionales con pensamiento crítico para que solucionen problemas, por lo cual se deben utilizar estrategias didácticas que permitan aprender una disciplina y determinar la utilidad en el contexto, haciendo que los estudiantes sean protagonistas en la construcción de su propio conocimiento, en la medida en que articulan habilidades para actuar de manera dinámica en los procesos escolares y sociales.

\section{Consideraciones finales}

Los estudiantes reconocieron la importancia de abordar la cuestión sociocientífica de los xenobióticos, estableciendo relaciones entre conceptos bioquímicos y evaluación de casos clínicos en su profesión.

Teniendo en cuenta la importancia de la ciencia y la tecnología en cuestiones sociales y ambientales de la sociedad actual, es con- veniente que los futuros profesionales de la salud tengan la capacidad de formarse como ciudadanos responsables con pensamiento crítico, para profundizar en un tema específico y para solucionar diferentes situaciones polémicas en contexto. Las herramientas que los docentes brindan a sus estudiantes en el proceso de enseñanza son fundamentales para el desarrollo sociocultural de los mismos, de ahí, la importancia de desarrollar habilidades que permitan ampliar el pensamiento crítico.

Precisamente el trabajo desarrollado, a partir la cuestión sociocientífica de los xenobióticos, también posibilitó articular aspectos conceptuales de la bioquímica con sus implicaciones socioambientales, favoreciendo el desarrollo de la habilidad de pensamiento crítico de solucionar problemasy análisis de argumentos. Estas habilidades permitieron que los estudiantes explicaran de manera coherente diversos cuestionamientos realizados en el grupo de estudio, utilizando el lenguaje de la bioquímica y la influencia de esta disciplina en la sociedad, se comprendió además como la bioquímica es útil en diversos aspectos de la cotidianidad, por lo que permite generar personas más analíticas que promueven soluciones a diversas situaciones relacionadas con el profesional en fisioterapia.

Esta estrategia didáctica es una alternativa a la tradicional memorización de términos y procesos bioquímicos sin aplicabilidad, permitiendo que los estudiantes reflexionen y critiquen diversas situaciones problemas enmarcadas en la situación controvertida, por lo que promovió la necesidad en los estudiantes de formarse como personas más críticas que propendan por el mejoramiento de la calidad de vida propia y de otras personas.

El desarrollo de habilidades de pensamiento crítico de solucionar problemas y análisis de argumento es posible cuando se 
Desarrollo de pensamiento crítico en estudiantes de Fisioterapia, a partir del estudio de las implicaciones sociocientíficas de los xenobióticos Nidia Yaneth Torres Merchán | Leonardo Fabio Martínez Pérez

presenta a los estudiantes situaciones cotidianas y controvertidas que exijan muchas formas de consideración de manera integrada, lo cual permite a los estudiantes reflexionar, debatir y explicar los diversos posicionamientos.

El trabajo de casos clínicos con cuestiones sociocientíficas permitió evidenciar el aprendizaje de los conceptos bioquímicos asumiendo que la formación de un profesional depende de la manera como la información es asimilada, interrelacionada, trabajada y generalizada. Se debe enseñar a partir de los problemas que el fisioterapeuta deberá resolver. El trabajo de cuestiones sociocientíficas tiene potencialidades para desarrollar una actitud crítica de investigación en los estudiantes, estableciendo una coherencia lógica entre teoría y práctica, proporcionando al alumno capacidades para interpretar datos y utilizar habilidades para resolver problemas que se presenten en su campo profesional.

Finalmente, el abordaje de cuestiones sociocientíficas en la enseñanza de la bioquímica puede favorecer importantes reflexiones sobre la práctica docente y hacer un reposicionamiento de la autonomía profesional de los profesores de ciencias, transformando la enseñanza tradicional basada en la transmisión de contenidos por un proceso de enseñanza constructivo, formativo y crítico.

\section{Bibliografía}

Dillon, J. (1994). Using discussion in classrooms. Buckingham: Open University Press.

Denzin, N. y Lincoln, Y. (org). (2006). O Planejamento da pesquisa qualitativa: teorias e abordagens. $2^{\text {a }}$ ed. Porto Alegre: Artes médicas.
Facione, P. (1998). Critical thinking: What it is and why it counts. Millbrae, CA: California Academic Press.

Fernández. G. (2003). O Ensino de Bioquímica para o curso de Fisioterapia. Santa Catarina: Editorial USC.

Gómez A. (2000). Bases teóricas de una propuesta didáctica para favorecer la comunicación en el aula. En Jorba, J., Gómez, I. y Prat, A. Hablar y escribir para aprender. Uso de la lengua en situaciones de enseñanzaaprendizaje desde las áreas curriculares. Barcelona. ICE UAB. Síntesis.

Halpern, D. (1998). Teaching critical thinking for transfer across domains. American Psychologist, 53(4), pp. 449-455.

Henderson, J. and Knutton, S.(1990). Biotechnology in Schools, A Handbook for Teachers, Philadelphia: Open University Press, hal. pp. 38-55.

Izquierdo, M. y Sanmartí, N. (2000). Enseñar a leer y escribir textos de Ciencias Naturales. En Jorba, J., Gómez, I. y Prat, A. Hablar y escribir para aprender. Uso de la lengua en situaciones de enseñanza-aprendizaje desde las áreas curriculares. Barcelona. ICE UAB. Síntesis.

Kortland, K. (1996). An STS case study about students' decision making on the waste issue. Science Education, 80 (6) pp. 673689.

Lankshear, C., Gee, J. P., y Hull, G. (1996). The new work order: Behind the language of the necapitalism. Boulder, CO: Westview Press.

Martínez, L. (2010). A abordagem de questões sociocientíficas na formação continuada de profesores de Ciências: contribuições e dificuldades. Tesis de Doutorado: Facul- 
dade de Ciências da Universidade Estadual Paulista, Bauru. En línea: <http://www2. fc.unesp.br/BibliotecaVirtual/DetalhaDocumentoAction.do?idDocumento=325 > Consultado 10 de oct de 2010.

Pedretti, E. (2003). Teaching science, technology, society and Environment (STSE) education: Preservice Teachers' philosophical and pedagogical landscapes, en Zeidler, D. (eds). The role of moral reasoning on socioscientific issues and discourse in science education, pp. 219-239. The Netherlands: Kluwer Academic Publishers.

Rudduck, J. (1986). A stratgy for handling controversial issues in the secondary school. En: Wellinton J., Controversial issues in the curriculum, 6-18. Oxford: Basil Blacckwell.

Santos, W. y Mortimer, E. (2001). Tomada de decisão para ação social responsável no ensino de ciências. Ciência \& Educação, 7(1), pp. 95-111

Sadler, T. y Zeidler, D. (2004). The morality of socioscientific issues: construal and resolution of genetic engineering dilemmas. Science Education, 88 (1), pp. 4-27.

Saiz, C. y Nieto, A. (2002). Pensamiento crítico: capacidades y desarrollo. En: Saiz, C (Ed.) Pensamiento crítico: conceptos básicos y actividades prácticas. Madrid: Pirámide. pp. 15-19.

Stenhouse, L. (1970). The humanities Project: An introduction, London: Heinemann

Zeidler, D.; Walker, K.; Ackett,W. A. y Simmons, M. (2002). Tangled up in views: Beliefs in the nature of science and responses to socioscientific dilemmas. Science Education, 86 (3), pp. $343-367$. 


\section{Anexo 1}

Fragmento de test de HCTAES para habilidades de análisis de argumento y solución de problemas

\section{(1) Parte 1}

Se pidió a un candidato a la presidencia que explicara su posición acerca de una propuesta de ley para proporcionar agujas limpias a los drogadictos como medio para prevenir la propagación de epidemias como el sida. Contestó que se oponía al programa "agujas limpias" porque era un error.

En una frase, describe el proceso de pensamiento que revela el candidato en esta respuesta.

\section{(1) Parte 2}

Se pidió a un candidato a presidente de gobierno que explicara su posición acerca de una propuesta de ley para proporcionar agujas limpias a los drogadictos como medio para prevenir la propagación de epidemias como el sida. Contestó que se oponía al programa "agujas limpias" porque era un error.

¿Cuáles de las siguientes críticas al candidato son razonables? (Elige tantas como sean aplicables).

a. El candidato no deja claro si estaba a favor o en contra del programa "agujas limpias".

b. El candidato no aporta una buena razón para su decisión.

c. El candidato no da ninguna razón sobre su decisión.

d. El candidato va a incrementar la propagación de la enfermedad al haber drogadictos que utilicen agujas usadas.

d. El candidato usa una etiqueta en lugar de una razón.

e. El candidato no se preocupa por los drogodependientes. 
TEA Tecné, Episteme y Didaxis

No. $29 \cdot$ Primer semestre de $2011 \cdot$ pp. 65-84

\section{(2) Parte 1}

Si el gobierno está haciendo una buena labor, entonces el empleo y otros indicadores económicos reflejarán una economía fuerte. Los índices de empleo en este momento son mejores que nunca y la mayoría de los otros indicadores muestran que la economía está saneada.

\section{Basándote en esta información ¿puedes concluir algo acerca del tipo de trabajo que está realizando el gobierno?}

Sí.

No.

Por favor, explica tu respuesta.

\section{(2) Parte 2}

Si el gobierno está haciendo una buena labor, entonces el empleo y otros indicadores económicos reflejarán una economía fuerte. Los índices de empleo son, en este momento, mejores que nunca y la mayoría de los otros indicadores muestran que la economía está saneada.

\section{Basándote en esta información, elige la mejor respuesta de las siguientes.}

a. El gobierno debe de estar realizando un buen trabajo.

b. El gobierno debe de estar realizando un mal trabajo.

c. No hay una conclusión definitiva -el gobierno puede estar realizando o no un buen trabajo.

d. La tasa de empleo no está relacionada con otros indicadores del estado de la economía.

e. La tasa de empleo no está relacionada con el tipo de política que el gobierno está llevando a cabo.

\section{(3) Parte 1}

Hay muchas oportunidades para los especialistas en informática. La verdad es que deberías especializarte en esta ciencia. El trabajo es interesante, hay muchas posibilidades de empleo y los sueldos son buenos. Por supuesto, no es una buena especialidad si se te dan mal las matemáticas o te gusta trabajar al aire libre.

¿Cuál es la conclusión de este breve párrafo?

¿Cuáles son las razones que la apoyan? 
Desarrollo de pensamiento crítico en estudiantes de Fisioterapia, a partir del estudio de las implicaciones sociocientíficas de los xenobióticos Nidia Yaneth Torres Merchán | Leonardo Fabio Martínez Pérez

\section{(3) Parte 2}

Hay muchas oportunidades para los especialistas en informática. La verdad es que deberías especializarte en esta ciencia. El trabajo es interesante, hay muchas posibilidades de empleo y los sueldos son buenos. Por supuesto, no es una buena especialidad si se te dan mal las matemáticas o te gusta trabajar al aire libre.

Para cada una de las siguientes afirmaciones, indica (C) si es una conclusión, $(R)$ si es una razón o (CA) si es un contra argumento. Elige una respuesta para cada una.

\begin{tabular}{|c|c|c|c|c|}
\hline \multirow{2}{*}{\multicolumn{2}{|c|}{ Opciones }} & \multicolumn{3}{|l|}{ Respuesta: } \\
\hline & & C & $\mathrm{R}$ & $C A$ \\
\hline 1) & $\begin{array}{l}\text { Hay muchas oportunidades para } \\
\text { los especialistas en informática. }\end{array}$ & $\mathrm{O}$ & $\mathrm{O}$ & $\mathrm{O}$ \\
\hline 2) & $\begin{array}{l}\text { La verdad es que deberías } \\
\text { especializarte en esta ciencia. }\end{array}$ & $\mathrm{O}$ & 0 & $\mathrm{O}$ \\
\hline 3) & El trabajo es interesante. & $\mathrm{O}$ & 0 & $\mathrm{O}$ \\
\hline 4) & Los sueldos son buenos. & $\mathrm{O}$ & $\mathrm{O}$ & $\mathrm{O}$ \\
\hline 5) & $\begin{array}{l}\text { No es una buena especialidad si se } \\
\text { te dan mal las matemáticas. }\end{array}$ & $\mathrm{O}$ & $\mathrm{O}$ & $\mathrm{O}$ \\
\hline
\end{tabular}


TE $\Delta$ Tecné, Episteme y Didaxis

No. 29 • Primer semestre de $2011 \cdot$ pp. 65-84

\section{Anexo 2}

Preguntas realizadas en la entrevista.

1. ¿Cómo trabajaban en la clase de bioquímica antes de comenzar a estudiar la cuestión de los xenobióticos?

2. ¿Cómo era la metodología de trabajo en la clase de bioquímica?

3. ¿Qué piensas acerca del trabajo desarrollado sobre los xenobióticos?

4. ¿Cómo les pareció la metodología desarrollada en las clases de bioquímica para trabajar este tema?

4. ¿En qué les contribuyó este trabajo para su formación profesional?

5. ¿Qué piensan ahora de la clase de bioquímica?

6. Si ustedes fueran a evaluar un caso clínico relacionado con algún xenobiótico, ¿qué aspectos tendrían en cuenta para evaluar el caso?

7. ¿Cómo entienden ustedes la ciencia en la sociedad?

8. ¿Cómo entienden la tecnología en la sociedad?

9. ¿Cómo entienden o consideran la relación de la ciencia y la tecnología en la sociedad?

10. ¿Cómo entienden o consideran la relación de la ciencia y la tecnología con el ambiente?

11. ¿Los aspectos indicados por ustedes anteriormente fueron trabajados de alguna manera en las clases de bioquímica orientadas a discutir la cuestión o el tema de los xenobióticos? 